Europhys. Lett., 74 (1), pp. 151-155 (2006)

DOI: $10.1209 / \mathrm{epl} / \mathrm{i} 2005-10496-0$

\title{
The break-up of the vortex structure in a mesoscopic wire containing a constriction
}

\author{
A. K. Elmurodov ${ }^{1}$, D. Y. Vodolazov ${ }^{1,2}$ and F. M. Peeters ${ }^{1}$ \\ 1 Departement Fysica, Universiteit Antwerpen - Groenenborgerlaan 171 \\ B-2020 Antwerpen, Belgium \\ 2 Institute for Physics of Microstructures, Russian Academy of Sciences \\ 603950 Nizhny Novgorod, GSP-105, Russia
}

received 8 November 2005; accepted in final form 2 February 2006 published online 22 February 2006

PACS. 74.25.-q- Properties of type I and type II superconductors.

PACS. 74.25.Qt - Vortex lattices, flux pinning, flux creep.

PACS. 74.78.-w - Superconducting films and low-dimensional structures.

\begin{abstract}
Within the nonlinear Ginzburg-Landau theory, we study the superconducting state in a mesoscopic wire containing a narrow constriction in the presence of a uniform magnetic field directed along the wire. If the narrow region is small enough so that no vortices can penetrate through it, curved vortices are formed, i.e. they enter at the top of the sample (the widest part) and exit near the constriction. At high magnetic fields a giant vortex is nucleated in the widest part of the wire which breaks up into a smaller giant and/or individual vortices near the constriction.
\end{abstract}

Introduction. - In recent years flat mesoscopic superconducting (SC) structures have been studied intensively both experimentally [1] and theoretically [2]. Unexpected new physics was found in superconducting $\mathrm{Al}$ disks, where the qualitative behavior of the magnetization $(M)$ as a function of the magnetic field $(H)$ was a sensitive function of the disk radius $R$ : for very small radius of the sample the magnetization was continuous, for intermediate values of the disk radius a discontinuous $M-H$ behavior was observed with a single jump as in type-I $\mathrm{SC}$, and for larger radius of the disk multiple jumps are present in the magnetization, which is close to the behavior observed for bulk type-II superconductors. Note that bulk $\mathrm{Al}$ is a type-I superconductor. A theoretical investigation of the vortex structure in such disks based on the Ginzburg-Landau (GL) theory predicted [3] that in such disks the vortices can coalesce into a single giant vortex or into single vortices dependent on the relative size, i.e. $R / \xi(T)$, and applied magnetic field $(\xi(T)$ is the temperature-dependent coherence length). The transition between the giant to multi vortex state is of second order, as was recently confirmed experimentally [4].

The above systems are in essence two-dimensional (2D), i.e. the Cooper pair density does not vary along the thickness of the disk. The other limit of SC wires has also been investigated both theoretically [5] and experimentally [6]. As long as the wire is homogeneous the vortex structure will not vary along the wire and finding the spatial location of the vortices is a twodimensional (2D) problem. Also in this case giant and multi-vortex configuration have been predicted. Since the type of vortex state which nucleates depends crucially on the relative 


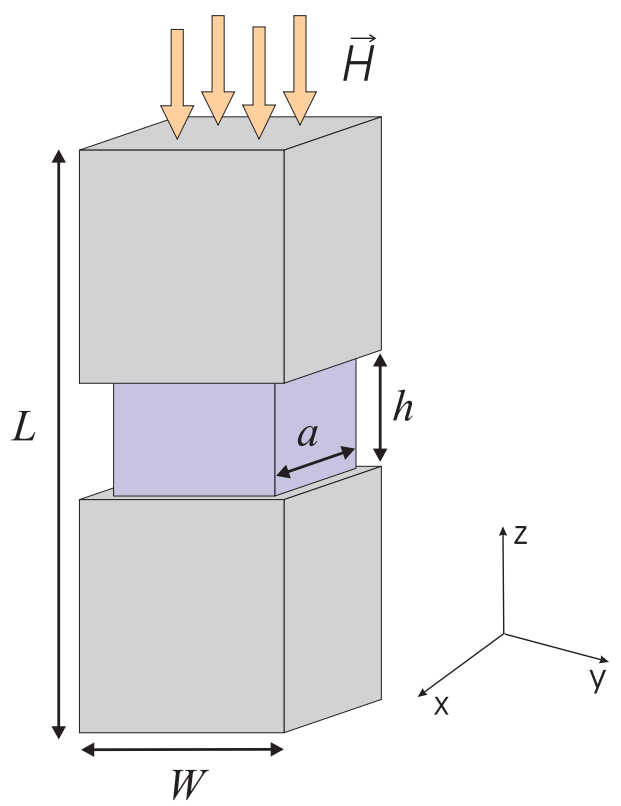

Fig. 1 - Schematic picture of the mesoscopic superconducting wire.

thickness of the wire, i.e. $R / \xi(T)$, one may wonder what will happen with the vortex structure in a SC wire with variable thickness. For example, at the region where the width is sufficiently large $N$ vortices penetrate the wire at a specific magnetic field, while in the narrow region of the wire only $N^{\prime}<N$ vortices can be stabilized. How will this transition from $N$ to $N^{\prime}$ vortices occur? Because the nucleation of a giant vortex state is very sensitive to the wire width, one can imagine that in a certain region of the wire one has a giant vortex while in a wider area of the wire multi-vortices are found. The splitting of a giant vortex into single vortices is a new phenomenon which has not been investigated up to now.

To address the above physical phenomena we consider a model SC square wire which contains locally a constriction (see fig. 1).

The system we will investigate is related to a recently fabricated device where transport through a core-shaped superconducting scanning tunnel microscope (STM) tip was investigated. [7-9]. In order to explain some of these experimental results, Misko et al. [10] considered a conoidal geometry. They found that superconductivity survives in the narrowest part of the $\mathrm{SC}$ at magnetic fields much larger than the critical field of the bulk material. This is a direct consequence of surface superconductivity which is enhanced at sharp corners or at small areas of the SC [11]. Unfortunately, their approach did not allow for states with nonzero vorticity. They assumed cylindrically symmetric solutions, which cannot give the correct vortex configuration in this geometry.

Theory. - We apply the nonlinear Ginzburg-Landau equations to describe the behavior of the order parameter as a function of the applied magnetic field, which is taken in the $z$-direction,

$$
(\nabla-i A)^{2} \psi+\left(1-|\psi|^{2}\right) \psi=0 .
$$

In eq. (1) distance is scaled in units of the temperature-dependent coherence length $\xi(T)$, the order parameter is in units of $\sqrt{\alpha / \beta}$, with $\alpha, \beta$ being the GL coefficients, and the magnetic field is in units of the second critical field $H_{c 2}(T)$. We used the boundary condition 
$\left.\left(-i \vec{\nabla}_{2 D}-\vec{A}\right) \Psi\right|_{n}=0$ at the edge of the wire and the superconducting normal metal boundary condition $\psi(0)=0, \psi(L)=0$ at the top and bottom of the sample.

The region of applicability of the stationary Ginzburg-Landau equation, eq. (1), is rather large, especially for "dirty" superconductors where the mean free path length $l$ is much smaller than the coherence length $\xi_{0} \sim v_{F} / \Delta(0)\left(\Delta_{0}\right.$ is the superconducting gap at $T=0$ and $v_{F}$ is the velocity of electrons at the Fermi surface). They are valid in the limit $\xi(0) \ll \xi(T)$ which is satisfied pretty well for $0.9 T_{c}<T<T_{c}$. Moreover, it was found that the formal application of the Ginzburg-Landau equations, when investigating the vortex structure in mesoscopic superconductors, gives quantitative agreement with experiment even for $T \rightarrow 0[2,4]$. Because the majority of microscopic and nanosize superconductors are in the "dirty" limit, we expect that our results are applicable over a large range of temperatures and sizes of the SC. We will also neglect screening effects which is a reasonable approximation for "dirty" superconductors where the London penetration depth $\lambda$ is much larger than the coherence length. Therefore, even for a sample with size of several $\xi$ the magnetic field inside the mesoscopic superconductor is approximately equal to the applied one and consequently we may neglect the second GL equation. Quantitatively this means that we chose $\xi<a \ll \lambda$ (see fig. 1).

To solve eq. (1) numerically we discretised the spatial coordinates and implemented the Euler method. The grid size was typically $0.5 \xi$ in all directions. Decreasing the grid step resulted only in small quantitative changes, but did not influence the qualitative features and the interesting physics which we found. As an example, we took in our calculations $L=50 \xi$, $W=6 \xi, a=4 \xi$ and $h=10 \xi$. A magnetic field is applied in the $z$-direction and the vector potential is expressed in the symmetric gauge $\boldsymbol{A}=(-H y / 2, H x / 2,0)$.

Results and discussion. - When we apply a magnetic field the order parameter is more strongly suppressed in the widest parts because the induced screening current $\boldsymbol{j}_{s}=-|\psi|^{2} \boldsymbol{A}$ is larger there than in the narrower part. The Meissner state becomes unstable for $H \simeq 0.6 H_{c 2}$ at which point two vortices enter the top, and bottom part of the SC wire while keeping the constriction free of vortices. Actually in our system the surface barrier effects are very strong,

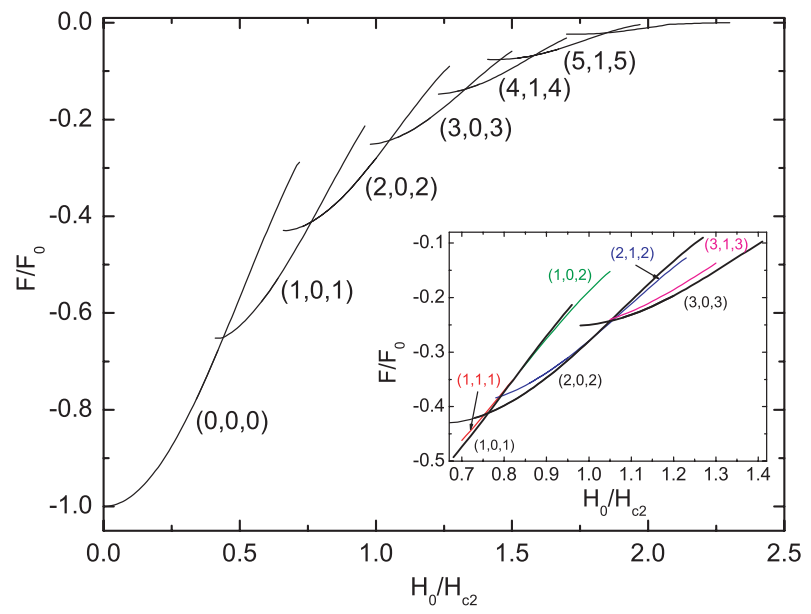

Fig. 2 - (Colour on-line) The free energy of a nanowire with a construction as a function of the applied magnetic field for $W=6 \xi, L=50 \xi, a=4 \xi, h=10 \xi$ (see fig. 1). The vortex state is characterized by three numbers: $\left(n_{t}, n_{c}, n_{b}\right)$ which refer, respectively, to the vorticity in the top, middle and bottom part of the wire (see fig. 1). The inset shows an enlargement of the intermediate magnetic-field region with the metastable states shown in colour. 


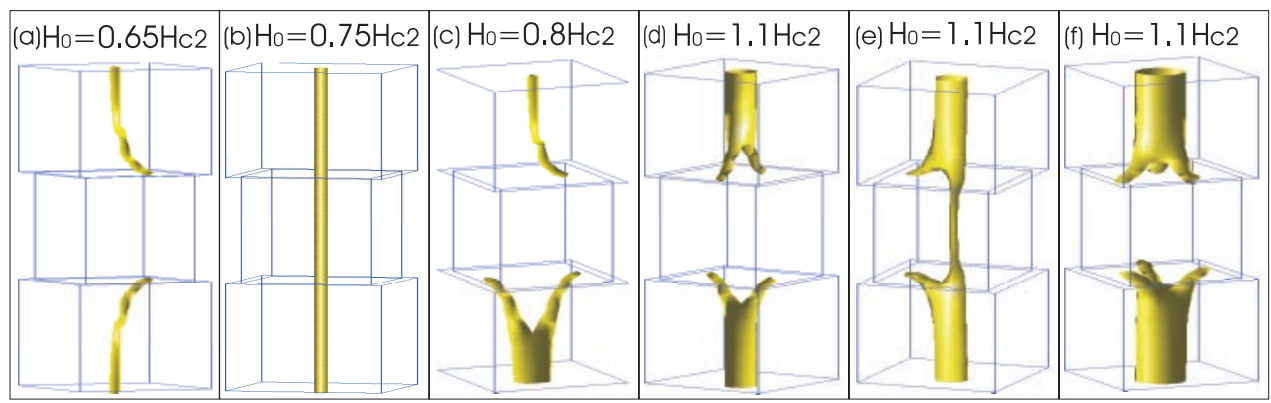

Fig. 3 - (Colour on-line) Three-dimensional isosurfaces of the Cooper-pair density at different magnetic fields and for different vortex configurations: (a) $(1,0,1)$, (b) $(1,1,1),(\mathrm{c})(1,0,2),(\mathrm{d})(2,0,2)$, (e) $(2,1,2)$ and (f) $(3,0,3)$.

which is the reason for the large stability range of the metastable states. In fig. 2 we plot the dependence of the free energy of our system for different vortex configurations where the vortex state is indicated as $\left(n_{t}, n_{c}, n_{b}\right)$, where $n_{t}\left(n_{c}, n_{b}\right)$ refers to the number of vortices in the top (constriction, bottom) area of the $\mathrm{SC}$ wire. The $3 \mathrm{D}$ contour plots of the corresponding order parameter (vortex) distribution are depicted in fig. 3 .

Notice that nonsymmetric vortex states, with respect to $z=0$ may exist in our system as metastable states. For example, the vortex state $(1,0,2)$ is metastable and has a higher energy than the symmetric vortex configuration (see inset in fig. 2). The surface barrier for vortex entry/exit is responsible for the strong hysteresis which is an intrinsic property of our wire. For example, when we sweep down the magnetic field it is quite difficult to expel a vortex from the construction when it is present. The barrier leads to the stability of such a state up to $H \simeq 0.7 H_{c 2}$ although energetically this state becomes unfavorable already at $H \simeq 1.3 H_{c 2}$.

Another interesting property of our system appears at large magnetic fields. At $H>$ $1.2 H_{c 2}$ the multi-vortex state can no longer be sustained in the wide parts of the wire and it transits to the giant-vortex state. When this giant vortex approaches the construction it splits into several vortices, because superconductivity is enhanced in the construction and the giant vortex is no longer stable (or metastable) here. As a result, the giant vortex splits into multi-vortices near the construction where some vortices exit the sample (see fig. 4). Near

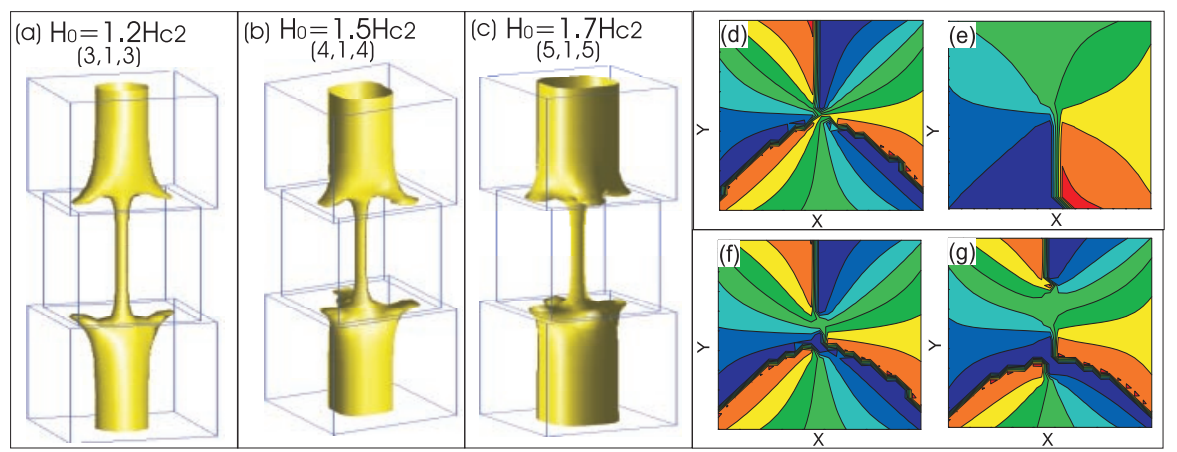

Fig. 4 - (Colour on-line) Three-dimensional isosurfaces of the suppressed Cooper-pair density in the region $H \geq 1.2 H_{c 2}$. Contour plots of the phase of the order parameter at the top (d), in the construction (e) and at the bottom of the wire are shown for the metastable vortex state $(3,1,3)$ for $H=1.3 H_{c 2}$ : (f) $z=7 \xi$ above the construction and (g) $z=0.5 \xi$ just above the constriction. 
the constriction, but still in the upper part of the wire, the giant vortex $L=3$ breaks up into a single vortex in the center and a $L=2$ giant vortex which is displaced towards the edge of the wire (see figs. $4(\mathrm{f}, \mathrm{g}))$. The latter two vortices move out of the system at the edge of the constriction. The contour plots of the phase for $H=1.3 H_{c 2}$ show that in the upper thick part of the wire (see fig. $4(\mathrm{~d})$ ) we have a giant vortex with vorticity $L=3$ and in the constriction (fig. 4(e)) only one vortex is present. This spatial break-up of the giant vortex state into multi-vortices has not been predicted before.

Conclusions. - In conclusion, we investigated vortex states in a nonuniform rectangular wire using the Ginzburg-Landau equation for the distribution of the order parameter (pairing potential of the Cooper pairs). Although we presented numerical results only for one specific set of parameters we found that the new physics is representative and typical for wires containing a local constriction. Namely, in a system where the constriction has a size less than or comparable to the coherence length, the vortex cannot enter the construction (there is not enough space for it). Therefore, vortices nucleated in the widest part of the sample will be forced to be curved and exit the sample before reaching the constriction. At high magnetic field it will lead to the splitting of the giant vortex into a smaller giant or a multi-vortex state in the constriction region. Because of the inevitable presence of a surface barrier the development of the vortex structure is a hysteretic process and it depends on the preceding history of the variation of the applied magnetic field. The entrance and exit of vortices in the constriction should be reflected in the magnetoresistance and the magnetization of the sample.

This work was supported by the Belgian Science Policy, the Flemish Science Foundation (FWO-Vl) and the ESF-network: AQDJJ. One of us (DYV) is supported by an INTAS Young Scientist Fellowship 04-83-3139.

\section{REFERENCES}

[1] Geim A. K., Grigorieva I. V., Dubonos S. V., Lok J. G. S., Mann J. C., Filippov A. E. and Peeters F. M., Nature (London), 390 (1997) 256.

[2] Singha Deo P., Schweigert V. A., Peeters F. M. and Geim A. K., Phys. Rev. Lett., 79 (1997) 4653.

[3] Schweigert V. A., Peeters F. M. and Singha Deo P., Phys. Rev. Lett., 81 (1998) 2783.

[4] Kanda A., Baelus B. J., Peeters F. M., Kadowaki K. and Oоtuka Y., Phys. Rev. Lett., 93 (2004) 257002.

[5] Stenuit G., Michotte S., Govaerts J. and Piraux L., Eur. Phys. J. B, 33 (2003) 103; Stenuit G., Michotte S., Govaerts J., Piraux L. and Bertrand D., Mod. Phys. Lett. B, 17 (2003) 537; Stenuit G., Michotte S., Govaerts J. and Piraux L., Supercond. Sci. Technol., 18 (2005) 174.

[6] Michotte S., Piraux L., Dubois S., Pailloux F., Stenuit G. and Govaerts J., Physica $C, \mathbf{3 7 7}(2002) 267$.

[7] Poza M., Bascones E., Rodrigo J. G., Agrait N., Vieira S. and Guinea F., Phys. Rev. $B, 58$ (1998) 11173.

[8] Suderow H., Bascones E., Izquierdo A., Guinea F. and Vieira S., Phys. Rev. B, 65 (2002) 100519(R).

[9] Rodrigo J. G., Suderow H. and Vieira S., Phys. Status Solidi B, 1 (2003) 386.

[10] Misko V. R., Fomin V. M. and Devreese J. T., Phys. Rev. B, 64 (2001) 014517.

[11] Schweigert V. A. and Peeters F. M., Phys. Rev. B, 60 (1999) 3084. 